\title{
The addition of reduced glutathione to cryopreservation media induces changes in the structure of motile subpopulations of frozen- thawed boar sperm
}

\author{
Efrén Estrada, Maria M. Rivera del Álamo, Joan E. Rodríguez-Gil, Marc Yeste* \\ Unit of Animal Reproduction, Department of Animal Medicine and Surgery, Faculty of Veterinary Medicine, Autonomous University of Barcelona, E-08193 \\ Bellaterra (Cerdanyola del Vallès), Barcelona, Spain
}

\section{A R T I C L E I N F O}

\section{Article history:}

Received 17 April 2017

Received in revised form

6 June 2017

Accepted 7 July 2017

Available online 8 July 2017

\section{Keywords:}

Pigs

Reduced glutathione

GSH

Spermatozoa

Cryopreservation

Motile sperm subpopulations

\begin{abstract}
A B S T R A C T
Adding cryopreservation media with reduced glutathione (GSH) has previously been shown to maintain the motility, membrane integrity and fertilizing ability of frozen-thawed boar sperm, although the effects of GSH on good (GFE) and poor freezability (PFE) ejaculates rely upon the intrinsic ejaculate freezability. The resilience to withstand freeze-thawing procedures has previously been related to the existence of a specific distribution of motile sperm subpopulations, which differs between GFE and PFE. Thus, the main aim of this study was to determine whether the addition of GSH to freezing media has any impact on the distribution of motile sperm subpopulations in GFE and PFE. With this purpose, 18 GFE and 13 PFE were cryopreserved with or without $2 \mathrm{mM} \mathrm{GSH}$. Sperm quality and motile subpopulations were evaluated at $30 \mathrm{~min}$ and $4 \mathrm{~h}$ post-thawing. Three subpopulations were identified and the percentages of spermatozoa belonging to the fastest and most linear subpopulation, which was referred as 'SP1', decreased over postthawing time. Good freezability ejaculates that were cryopreserved in the presence of $2 \mathrm{mM}$ exhibited a significantly higher percentage of spermatozoa belonging to SP1 than the other combinations of treatment and freezability both at $30 \mathrm{~min}$ (mean \pm SEM: GFE-C: $16.6 \pm 0.4$; GFE-GSH $27.7 \pm 0.6$ ) and 4 h postthawing (GFE-C: $7.8 \pm 0.2$ vs. GFE-GSH: $16.7 \pm 0.4$ ). In conclusion, the positive effect of GSH on the motility of frozen-thawed sperm is related to a specific sperm subpopulation (SP1), which could coincide with the fertile sperm one.
\end{abstract}

๑) 2017 Elsevier Inc. All rights reserved.

\section{Introduction}

Cryopreservation is known to impair boar spermatozoa (see Ref. [43] for review), but the extent of that cryodamage differs between boar ejaculates [37]. For this reason, ejaculates are classified as of good (GFE) or poor freezability (PFE) based upon their sperm quality at post-thawing [37,38].

Previous works attempted to increase the resilience of boar sperm to cryopreservation by adding antioxidants to freezing and thawing media (see Ref. [42] for review). Supplementing cryopreservation media with reduced L-glutathione (GSH) maintains better the integrity of nucleus and plasma membrane, and the fertilizing ability of frozen-thawed boar spermatozoa $[6,7,13,15,45]$.

\footnotetext{
* Corresponding author. Present address: Unit of Cell Biology, Department of Biology, Faculty of Sciences, University of Girona, C/ Maria Aurèlia Campany 69, E17003 Girona, Spain.

E-mail address: marc.yeste@udg.edu (M. Yeste).
}

Interestingly, the GSH-positive effect differs between ejaculates, so that the improvement mediated by this antioxidant is more apparent in GFE than in PFE [47]. However, while previous works on the effects of GSH upon cryopreserved boar sperm assessed different sperm parameters, no study has investigated whether this antioxidant affects the structure of motile sperm subpopulations.

Abaigar et al. [2] were the first to use advanced statistical methods for the analysis of Computer Assisted Sperm Analysis system (CASA) data. Following this landmark paper, motile sperm subpopulations have been identified in the ejaculates of separate mammalian species and have been suggested to have a functional role [33]. It is worth noting that sperm motile subpopulations rely on the kinetic characteristics of single sperm cells, which depend on their surrounding environment.

The number of sperm subpopulations varies across species and studies. For example, Santolaria et al. [34] identified three motile subpopulations in the sheep, whilst Luna et al. [19] identified four. Three motile sperm subpopulations were identified in the goat 
[39], and four in rabbits, donkeys and dogs [5,11,21-23,27,32]. In bulls, four sperm subpopulations were distinguished [25], the fastest one showing positive correlation with sperm binding to the zona pellucida, percentages of penetration and rates of pronucleus formation [9]. While Quintero-Moreno et al. [30] described four subpopulations in refrigerated stallion sperm, Ortega-Ferrusola et al. [26] found six and four subpopulations in fresh and frozenthawed stallion sperm, respectively. In boars, whilst some reports identified three motile sperm subpopulations $[2,4,8]$, others found four $[11,12]$. However, it is worth mentioning that the number and characteristics of those sperm subpopulations heavily relies upon the statistical method.

While the relevance of studying the structure of sperm subpopulations during freeze-thawing procedures of boar spermatozoa has been previously reported $[4,12,36]$, the effects of GSH on that subpopulation structure warrant further research. In addition, as GSH-effects depend on the intrinsic ejaculate freezability [47], it would be interesting to address whether the extent of changes in the motile subpopulations of frozen-thawed boar sperm induced by GSH differs between GFE and PFE. Therefore, the present study aimed at investigating the structure of motile subpopulations in frozen-thawed boar sperm of GFE and PFE, following supplementation of cryopreservation media with or without $2 \mathrm{mM} \mathrm{GSH}$.

\section{Materials and methods}

\subsection{Semen samples}

The current study involved a total of 36 ejaculates, which came from 25 separate boars. The ejaculates were obtained from a local farm (Selección Batallé, S.A.; Riudarenes, Spain), where animals were kept under adjusted conditions of temperature and humidity and were fed a standard diet with water provided ad libitum. These boars were routinely used for producing and selling semen doses for artificial insemination (AI). No fertility problems for these boars were recorded by the AI station. Ejaculates were collected twice a week through the gloved-hand technique [16]. Briefly, the spermrich fraction was collected and filtered through gauze and an insulated container containing $50 \mathrm{~mL}$ pre-warmed commercial extender (Duragen, Magapor, S.L.; Zaragoza, Spain) was used. After collection, the diluted sperm-rich fraction was re-diluted $1: 1(\mathrm{v} / \mathrm{v})$ with the same extender (Duragen, Magapor, S.L.) and cooled down to $17^{\circ} \mathrm{C}$. Ejaculates were transported to the laboratory within $4 \mathrm{~h}$ post-collection.

Upon arrival, all semen samples were evaluated and sperm quality parameters were confirmed to be above the following standard thresholds in 31 out of 36 ejaculates: viable spermatozoa $\geq 85 \%$; morphologically normal spermatozoa $\geq 85 \%$; total motile spermatozoa $\geq 80 \%$. The five ejaculates that did not satisfy the quality standards were excluded from the study and the other 31 were cryopreserved as described below.

The current study was designed following the Animal Welfare Directive issued by the Regional Government of Catalonia, Spain (D 214/1997, DOGC 1997; 2450: 9169-9174) and the Spanish welfare and protection standards in swine (RD 1392/2012, BOE 2012; 241: 71380-71382).

\subsection{Sperm cryopreservation}

Prior to cryopreservation, all ejaculates were stored for a 24 hperiod at $17{ }^{\circ} \mathrm{C}$ as this holding time has been reported to increase sperm survival at post-thawing [48]. Next, ejaculates were split into separate falcon tubes of $50-\mathrm{mL}$ each and then centrifuged at $17{ }^{\circ} \mathrm{C}$ and $600 \times \mathrm{g}$ for $5 \mathrm{~min}$. The resulting pellets were re-suspended in freezing medium (LEY) made up of $80 \%(\mathrm{v} / \mathrm{v}) 310 \mathrm{mM} \beta$-lactose
(Sigma-Aldrich ${ }^{\circledR}$; St Louis, MO, USA), 20\% (v/v) egg yolk, and $100 \mu \mathrm{g} \mathrm{mL} \mathrm{m}^{-1}$ kanamycin sulphate (Gibco ${ }^{\circledR}$; Life Technologies, Carlsbad, CA, USA). Final concentration, which was $1.5 \times 10^{9}$ spermatozoa $\cdot \mathrm{mL}^{-1}$ at this step, was adjusted with LEY medium after determining sperm concentration with a Makler counting chamber (Sefi-Medical Instruments; Haifa, Israel). The resulting volume was split into two fractions of equal volume. One of these fractions (referred as FT-GSH) was supplemented with GSH $\left(\mathrm{C}_{10} \mathrm{H}_{17} \mathrm{~N}_{3} \mathrm{O}_{6} \mathrm{~S}\right.$; Sigma-Aldrich ${ }^{\circledR}$; final concentration: $\left.2 \mathrm{mM}\right)$, whereas the other (called FT-Control) did not contain GSH (FTControl). The two aliquots were frozen in parallel. Spermatozoa were cooled from $17{ }^{\circ} \mathrm{C}$ to $5{ }^{\circ} \mathrm{C}$ at a rate of $-0.1^{\circ} \mathrm{C} \cdot \mathrm{min}^{-1}$ through a controlled-rate freezer (Icecube14S-B; Minitüb GmbH, Tiefenbach, Germany). Spermatozoa were re-diluted to a final concentration of $1 \times 10^{9}$ spermatozoa $\cdot \mathrm{mL}^{-1}$ with LEYGO medium, which consisted of LEY medium containing a cryoprotectant (glycerol; SigmaAldrich $^{\circledR}$ ) and a surfactant (Orvus ES Paste; OEP, Equex STM; Nova Chemical Sales Inc., Scituate, MA, USA). After dilution, final concentrations of glycerol and OEP within straws were $2 \%$ and $0.5 \%$, respectively. The LEYGO medium added to the GSH-supplemented aliquot also contained $2 \mathrm{mM} \mathrm{GSH}$. Following this, the volume of each aliquot was packed in separate $0.5-\mathrm{mL}$ plastic straws (Minitüb $\mathrm{GmbH}$ ). Each straw was labeled using an automatic printer for plastic straws (Easycoder; Minitüb $\mathrm{GmbH}$ ) with the following information: boar, ejaculate code, freezing date and treatment (FT-C or FT-GSH). In order to avoid any disturbance in the temperature of sperm samples, straws were cooled to $5{ }^{\circ} \mathrm{C}$ prior to sperm packaging. Upon sperm packaging, straws were transferred to the same controlled-rate freezer (Icecube14S-B; Minitüb $\mathrm{GmbH}$ ) used for cooling the samples. A freezing curve specifically designed for boar sperm was run and cooling rates and times were as follows: $\quad-6 \quad{ }^{\circ} \mathrm{C} \cdot \mathrm{min}^{-1}$ for 100 s $\quad$ (from $5 \quad{ }^{\circ} \mathrm{C}$ to $-5{ }^{\circ} \mathrm{C}$ ); $-39.82{ }^{\circ} \mathrm{C} \cdot \mathrm{min}^{-1}$ for $113 \mathrm{~s}$ (from $-5{ }^{\circ} \mathrm{C}$ to $-80{ }^{\circ} \mathrm{C}$ ), holding step at $-80{ }^{\circ} \mathrm{C}$ for $30 \mathrm{~s}$, and $-60{ }^{\circ} \mathrm{C} \cdot \mathrm{min}^{-1}$ rate for $70 \mathrm{~s}$ (from $-80{ }^{\circ} \mathrm{C}$ to $-150^{\circ} \mathrm{C}$ ). Straws were then plunged into liquid $\mathrm{N}_{2}$ and evaluated within the next two months.

For thawing, and as recommended by Casas et al. [3], four straws per treatment (FT-C or FT-GSH) and ejaculate were taken and shaken vigorously in a water bath at $37^{\circ} \mathrm{C}$ for $20 \mathrm{~s}$ [10]. The volume contained in the four straws was diluted with three volumes of Beltsville Thawing Solution (BTS; [29]) previously warmed at $37^{\circ} \mathrm{C}$. Sperm motility and membrane integrity were evaluated at $30 \mathrm{~min}$ and $4 \mathrm{~h}$ post-thawing, as $4 \mathrm{~h}$ is the insemination-to-ovulation interval recommended for cryopreserved boar sperm [40].

\subsection{Evaluation of sperm concentration and morphology}

Sperm concentration was only assessed when ejaculates were received in our laboratory and during cryopreservation steps (i.e. dilution in LEY, dilution in LEYGO, and at post-thawing). Briefly, three replicates were evaluated using a Makler counting chamber (Sefi-Medical Instruments) after dilution with $4 \%$ formalin buffered solution. Sperm count was adjusted to the dilution factor.

Sperm morphology was only assessed upon arrival of ejaculates in our laboratory in order to ensure that percentages of morphologically normal spermatozoa were above the threshold (85\%) set as a quality standard. Sperm cells were previously fixed with $4 \%$ paraformaldehyde (Sigma-Aldrich ${ }^{\circledR}$ ) and a drop of $5 \mu \mathrm{L}$ was placed onto a slide and then mounted with a cover slip. Samples were evaluated under a phase-contrast microscope (Olympus BX41) at $200 \times$ magnification (Olympus $20 \times 0.40$ PLAN objective lens, positive phase-contrast field) and three counts of 100 sperm each were made prior to calculating the mean \pm standard error of the mean (SEM). Each spermatozoon was classified into one of the following categories: morphologically normal, with proximal 
droplets, with distal droplets or aberrant (coiled tails, tails folded at the connecting piece, at the intermediate piece or at Jensen's ring).

\subsection{Evaluation of plasma membrane integrity}

The integrity of sperm plasma membrane was evaluated before cryopreservation and at $30 \mathrm{~min}$ and $4 \mathrm{~h}$ post-thawing using a flow cytometer (Cell Laboratory QuantaSC ${ }^{\text {тм }}$ cytometer (Beckman Coulter; Fullerton, CA, USA) and following staining with SYBR14 and propidium iodide (PI; [14]). Before evaluation, sperm were diluted with BTS at a final concentration of $1 \times 10^{6}$ spermatozoa $\cdot \mathrm{mL}^{-1}$ in a volume of $0.5 \mathrm{~mL}$. The equipment was calibrated periodically calibrated using $10-\mu \mathrm{m}$ Flow-Check fluorospheres (Beckman Coulter) and each sample was evaluated in triplicate using three independent tubes.

Briefly, spermatozoa were stained with SYBR14 (final concentration: $100 \mathrm{nM}$ ) and incubated at $37.5^{\circ} \mathrm{C}$ for $10 \mathrm{~min}$. Following this, samples were stained with PI (final concentration: $12 \mu \mathrm{M}$ ) and incubated at $37.5{ }^{\circ} \mathrm{C}$ for $5 \mathrm{~min}$. Spermatozoa were then evaluated with the flow cytometer, which excited the samples with an argon ion laser $(488 \mathrm{~nm})$ set at a power of $22 \mathrm{~mW}$. The sheath flow rate was set at $4.17 \mu \mathrm{L} \mathrm{min}{ }^{-1}$ and two filters (FL1 and FL3) were used to detect the fluorescence emitted by SYBR14 (FL-1: Dichroic/Splitter, DRLP: $550 \mathrm{~nm}$, Band Pass filter: $525 \mathrm{~nm}$, detection width 505-545 nm) and PI (FL-3: Long Pass filter: 670/30 nm). Electronic volume (EV) and side scatter (SS) were recorded in linear mode (EV vs. SS dot plots) for a total of 10,000 events per sample. Spermspecific events were gated on the basis of EV/SS distributions, and EV channel was adjusted to exclude subcellular debris (particle diameter $<7 \mu \mathrm{m}$ ) and cell aggregates (particle diameter $>12 \mu \mathrm{m}$ ). Dot-plots (FL1 vs. FL3) allowed distinguishing viable spermatozoa (i.e. with an intact plasma membrane; SYBR14 ${ }^{+} / \mathrm{PI}^{-}$) from nonviable spermatozoa (SYBR14 $/ \mathrm{PI}^{+}$and $\mathrm{SYBR} 14^{+} / \mathrm{PI}^{+}$) and nonDNA-containing particles (debris; SYBR14 $/ \mathrm{PI}^{-}$). Spill-over of green fluorescence (SYBR14) was compensated into FL-3 channel (2.45\%).

\subsection{Evaluation of sperm motility}

Sperm motility was evaluated before cryopreservation and at $30 \mathrm{~min}$ and $4 \mathrm{~h}$ post-thawing using a computer assisted sperm analysis (CASA) system (Sperm Class Analyzer software; $\mathrm{SCA}^{\circledR} 5$, Microptic S.L.; Barcelona, Spain) and a phase contrast microscope (Olympus BX41) at $100 \times$ magnifications (negative phase contrast: Olympus $10 \times 0.30$ PLAN objective lens). Briefly, $20 \mu \mathrm{L}$ of each semen sample was placed on a Makler counting chamber (SefiMedical Instruments) pre-warmed at $37{ }^{\circ} \mathrm{C}$ and the following sperm parameters were evaluated [24]: total and progressive sperm motility; curvilinear velocity (VCL, $\mu \mathrm{m} \cdot \mathrm{s}^{-1}$ ), which consists of the average velocity measured over the actual point-to-point track followed by the cell; straight line velocity (VSL, $\mu \mathrm{m} \cdot \mathrm{s}^{-1}$ ), which is the average velocity measured in a straight line from the beginning to the end of a track; average path velocity (VAP, $\mu \mathrm{m} \cdot \mathrm{s}^{-1}$ ), which corresponds to the average velocity of the smoothed cell's pathway; linearity (LIN, \%), which consists of the quotient between VSL and VCL; straightness (STR, \%), which results from dividing VSL by VAP; motility parameter wobble (WOB, \%), which is the degree of oscillation of the actual sperm-head trajectory around its average path and is provided by the quotient between VAP and VCL; amplitude of lateral head displacement (ALH, $\mu \mathrm{m})$; and beat cross frequency (BCF, $\mathrm{Hz}$ ), which is the frequency at which the sperm cell's head crosses the sperm cell's average pathway. A sperm cell was considered to be motile when VAP was higher than $10 \mu \mathrm{m} \mathrm{s}^{-1}$. Three replicates were assessed per sample and at least 1000 spermatozoa were evaluated per replicate. For each assessment, overall and individual data (i.e. particular kinematic parameters for each sperm cell) were recorded.

\subsection{Statistical analyses}

Analyses of results were conducted using a statistical package $\left(\right.$ IBM $^{\circledR}$ SPSS $^{\circledR} 21.0$ for Windows; IBM Corp.; Chicago, IL, USA). Data were checked for normality (Shapiro-Wilk test) and homogeneity of variances (Levene test). The level of significance was set at $P \leq 0.05$ in all cases.

\subsubsection{Classification of ejaculates into groups of good (GFE) and poor freezability (PFE)}

Ejaculates were classified into two groups of freezability (i.e. resilience to withstand cryopreservation; GFE and PFE) according to their total sperm motility and membrane integrity $\left(\mathrm{SYBR} 14^{+} / \mathrm{PI}^{-}\right)$ determined at $30 \mathrm{~min}$ and $4 \mathrm{~h}$ post-thawing in control samples (FTC). With this purpose, separate cluster analyses (30 min and $4 \mathrm{~h}$ post-thawing) based on the between-groups linkage method and the squared Euclidean distances were run with a maximum number of two clusters. All ejaculates classified as GFE presented a minimum of $45 \%$ viable spermatozoa at 30 min post-thawing and a minimum of $30 \%$ viable spermatozoa at $4 \mathrm{~h}$ post-thawing.

The effects of supplementing freezing media with $2 \mathrm{mM}$ GSH on the motility and membrane integrity of frozen-thawed sperm were compared between GFE and PFE through a linear mixed model followed by a Sidak's post-hoc test. Post-thawing time (30 min and $4 \mathrm{~h}$ ) was the intrasubject factor, the ejaculate was the randomeffects factor and the treatment (control or GSH) and the freezability group (GFE or PFE) were the fixed-effects factors. All sperm parameters were considered as dependent variables.

\subsubsection{Determination of sperm subpopulations}

Sperm subpopulations were set according to the procedure described by Luna et al. [19] with minor modifications. In brief, the individual kinematic parameters obtained for each spermatozoon after CASA assessment (VSL, VCL, VAP, \%LIN, \%STR, \%WOB, ALH and $\mathrm{BCF}$ ) were used as independent variables in a Principal Component Analysis (PCA). These kinematic parameters were sorted into PCA components and the obtained data matrix was rotated using the Varimax procedure with the Kaiser normalization. As a result, regression scores for each of the PCA components were calculated per spermatozoon.

Cluster analyses were conducted using regression scores from all evaluations made for FT-C and FT-GSH at 30 min and 4 h postthawing. Sperm subpopulations were set through a two-step cluster analysis based upon log-likelihood distance and the Schwarz's Bayesian Criterion. A total of three motile sperm subpopulations were obtained. Proportions of sperm cells belonging to each subpopulation (SP1, SP2 or SP3) were calculated in each assessment (i.e. independent replicate consisting of a combination between boar ejaculate, treatment and post-thawing time).

The effects of supplementing cryopreservation media with $2 \mathrm{mM} \mathrm{GSH}$ on the distribution of sperm subpopulations at $30 \mathrm{~min}$ and $4 \mathrm{~h}$ post-thawing were compared between GFE and PFE through a linear mixed model followed by a Sidak's post-hoc test. In this model, the post-thawing incubation time was the intrasubject factor, the treatment (FT-C, FT-GSH) and the freezability group (GFE and PFE) were the fixed-effects factors and the ejaculate was 
considered the random-effects factor. Percentages of SP1, SP2 and SP3 spermatozoa were considered as dependent variables.

\section{Results}

\subsection{Classification of boar ejaculates}

As previously stated 31 out of the 36 ejaculates received were found to be above the sperm quality standards and were thus included in the study. From these 31 ejaculates, 18 were found to be GFE and the other 13 were classified as PFE. Classifications resulting from assessments at $30 \mathrm{~min}$ and $4 \mathrm{~h}$ post-thawing coincided (Supplementary Table 1).

\subsection{Effects of GSH on sperm membrane integrity and motility in GFE and PFE}

Fig. 1 shows the percentages of membrane-intact spermatozoa $\left(\mathrm{SYBR} 14^{+} / \mathrm{PI}^{-}\right.$) at $30 \mathrm{~min}$ and $4 \mathrm{~h}$ post-thawing in control (FT-C) and treatment (FT-GSH) for GFE and PFE. Percentages of SYBR14 ${ }^{+} / \mathrm{PI}^{-}$ spermatozoa were significantly $(P<0.05)$ higher in GFE than in PFE at $30 \mathrm{~min}$ and $4 \mathrm{~h}$ post-thawing, and differences between GFE and PFE were more apparent in FT-GSH than in FT-C. Although there was a significant $(P<0.05)$ reduction in the percentages of SYBR $14^{+} / \mathrm{PI}^{-}$spermatozoa over post-thawing incubation time (30 min vs. $4 \mathrm{~h}$ ), the treatments containing $2 \mathrm{mM} \mathrm{GSH}$ exhibited better sperm membrane integrity both at $30 \mathrm{~min}$ and $4 \mathrm{~h}$, especially in the case of GFE.

Percentages of total motile spermatozoa were similar to those observed for membrane-intact spermatozoa (Fig. 2). In effect, as expected, GFE showed significantly $(P<0.05)$ higher percentages of total motile spermatozoa than PFE and there was a significant $(P<0.05)$ reduction in sperm motility over post-thawing incubation time. Reduced glutathione was observed to maintain better the post-thaw sperm motility both at $30 \mathrm{~min}$ and $4 \mathrm{~h}$ post-thawing in GFE and PFE, although the extent of that improvement was higher in the former than in the latter.

\subsection{Principal component analysis and determination of sperm subpopulations}

Principal component analyses sorted the eight kinematic parameters (VCL, VSL, VAP, \%LIN, \%STR, \%WOB, ALH and BCF) into two main components (Table 1). The first component explained $63.64 \%$ variance and showed the highest square loading factors $\left(\mathrm{a}_{\mathrm{ij}}^{2}\right)$ for \% LIN, \%STR, \%WOB and VSL. The second component explained $21.21 \%$ variance and showed the highest $\mathrm{a}_{\mathrm{ij}}^{2}$ for VCL, ALH, VAP and BCF.

Regression scores obtained after PCA were used to cluster sperm cells and three different motile subpopulations were found. Table 2 shows the kinematic sperm parameters for SP1, SP2 and SP3. Subpopulation 1 was the fastest and most linear, showing the highest values for all kinematic parameters (i.e. VCL, VSL, VAP, \%LIN, \%STR, \%WOB, ALH and BCF). Subpopulation 2 was characterized by intermediate values, exhibiting lower kinematic parameters than SP1 but higher than SP3. Finally, SP3 included the slowest and nonlinear spermatozoa.

\subsection{Effects of GSH on distribution of sperm subpopulations in GFE} and PFE

Fig. 3 shows the proportions of spermatozoa belonging to SP1. In all combinations the percentages of SP1-spermatozoa significantly $(P<0.05)$ decreased over post-thawing time. However, GFE samples that had been cryopreserved in the presence of $2 \mathrm{mM} \mathrm{GSH}$ exhibited significantly $(P<0.05)$ higher percentages of SP1spermatozoa than the other combinations of treatment and freezability group both at 30 min (mean \pm SEM: GFE-C: $16.6 \pm 0.4$ vs. GFE-GSH $27.7 \pm 0.6 ; P<0.05$ ) and $4 \mathrm{~h}$ post-thawing (GFE-C: $7.8 \pm 0.2$ vs. GFE-GSH: $16.7 \pm 0.4 ; P<0.05$ ). The addition of $2 \mathrm{mM}$ GSH to freezing media increased the proportions of SP1spermatozoa in both GFE and PFE. Interestingly, PFE cryopreserved with $2 \mathrm{mM}$ GSH presented similar proportions of SP1spermatozoa than those GFE that did not contain GSH (30 min: PFE-GSH: $18.8 \pm 0.5$ vs. GFE-C: $16.6 \pm 0.4 ; P>0.05 ; 4$ h: PFE-GSH: $8.4 \pm 0.2$ vs. GFE-C: $7.8 \pm 0.2 ; P>0.05)$.

Proportions of spermatozoa belonging to SP2 are shown in

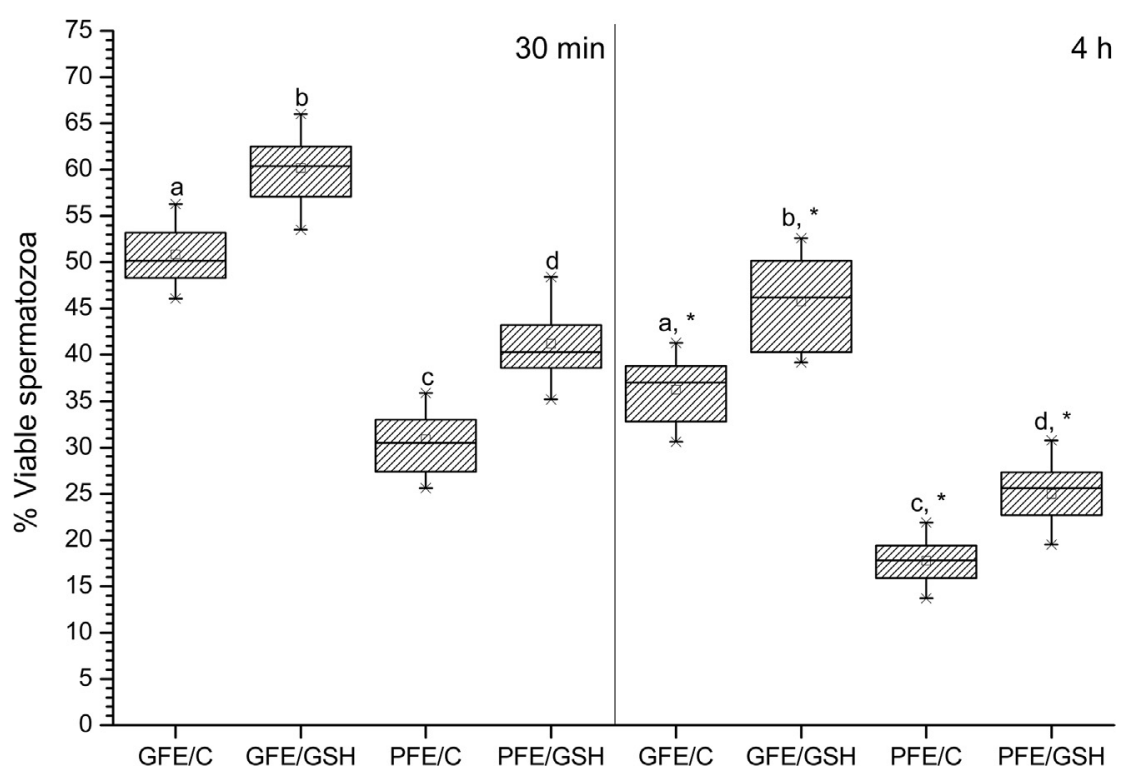

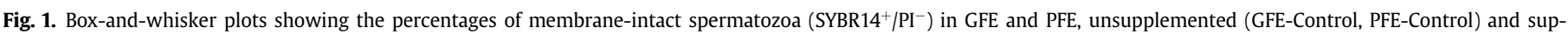

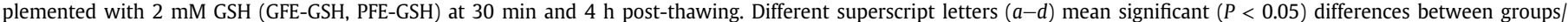

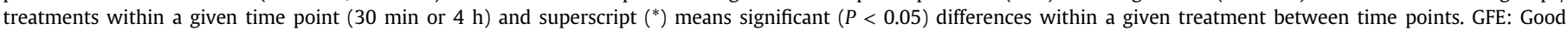
freezability ejaculates. PFE: Poor freezability ejaculates. GSH: Reduced glutathione (2 mM). 


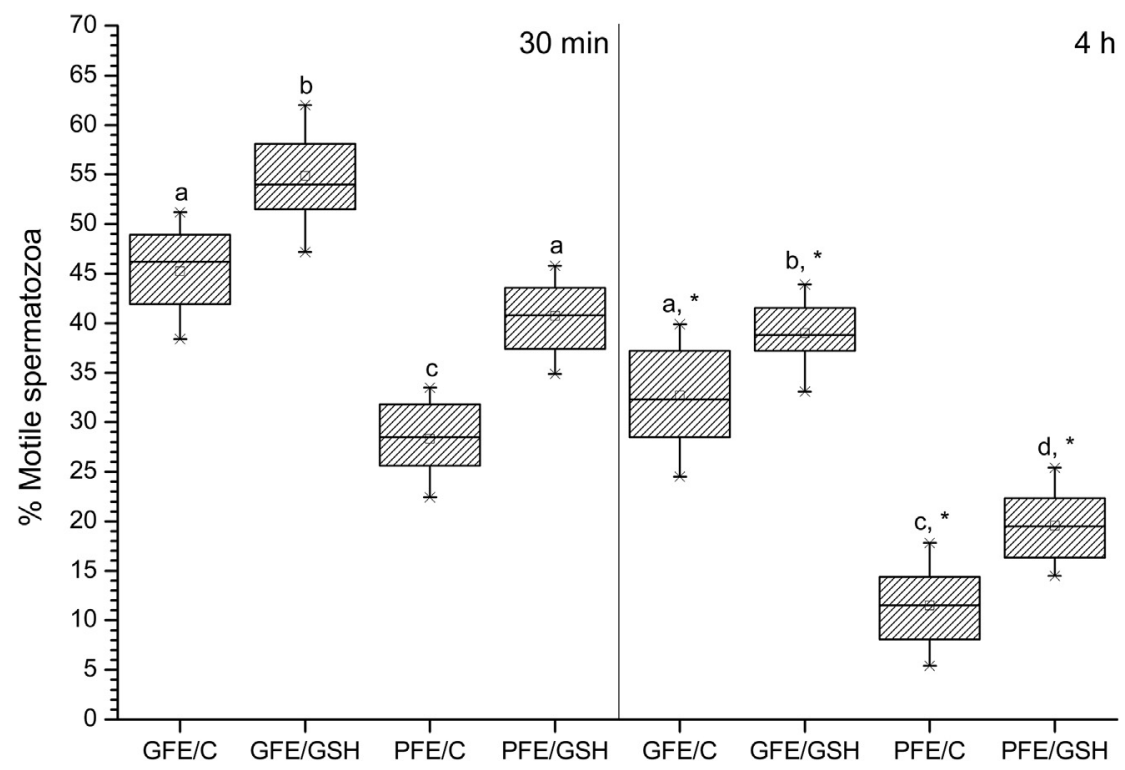

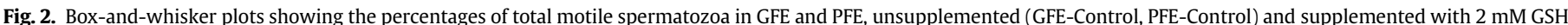

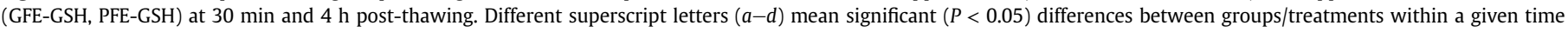

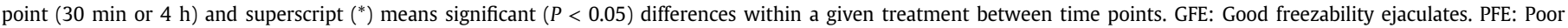
freezability ejaculates. GSH: Reduced glutathione (2 mM).

Table 1

Principal Component Analyses using the kinematic parameters (VCL, VSL, VAP, \%LIN, \%STR, \%WOB, ALH and BCF) of all individual sperm cells. As a result, two PCA components were obtained. $\mathrm{a}_{\mathrm{ij}}^{2}$ : square loading factors.

\begin{tabular}{llll}
\hline Component & Variance & Combinations of variable & $\mathrm{a}_{\mathrm{ij}}^{2}$ \\
\hline 1 & $63.64 \%$ & \%LIN & 0.95 \\
& & \%STR & 0.75 \\
& & \%WOB & 0.71 \\
2 & VSL & 0.55 \\
& \multirow{2}{*}{$21.21 \%$} & VCL & 0.89 \\
& & ALH & 0.82 \\
& & VAP & 0.60 \\
Total & & BCF & 0.40 \\
\hline
\end{tabular}

Fig. 4. It is worth noting that proportions of SP2-spermatozoa significantly decreased over post-thawing incubation time in all cases. At 30 min post-thawing, PFE cryopreserved without GSH showed a significantly $(P<0.05)$ lower percentage of spermatozoa belonging to SP2 than the other combinations of treatment and ejaculate freezability. In contrast, no effects of GSH-addition were seen either in GFE or in PFE at $4 \mathrm{~h}$ post-thawing.

With regard to proportions of spermatozoa belonging to SP3
(Fig. 5), post-thawing incubation time increased the percentages of sperm belonging to that motile subpopulation in all combinations of treatment and freezability group. However, GFE cryopreserved with GSH showed significantly $(P<0.05)$ lower percentages of SP3spermatozoa than those observed in all the other experimental groups both at $30 \mathrm{~min}$ and $4 \mathrm{~h}$ post-thawing. While PFE cryopreserved in the absence of GSH (PFE-Control) exhibited the highest percentages of SP3-spermatozoa (e.g. PFE-C $30 \mathrm{~min}$ : $60.6 \pm 0.5 v s$. GFE-C 30 min: $41.3 \pm 0.8 ; P<0.05)$, the presence of GSH in freezing media of PFE resulted in a reduction of that percentage, showing similar values to those of GFE-Control both at 30 min (PFE-GSH 30 min: $48.7 \pm 0.8$ vs. GFE-C 30 min: $41.3 \pm 0.8$; $P>0.05$ ) and $4 \mathrm{~h}$ post-thawing (PFE-GSH $4 \mathrm{~h}: 76.1 \pm 0.4 v s$. GFE-C 4 h: $72.2 \pm 0.6 ; P>0.05)$.

\section{Discussion}

The present study indicates that the improvement in the resilience of boar sperm to withstand cryopreservation induced by GSH is concomitant with a specific motile sperm subpopulational distribution in which the percentage of sperm with better motion characteristics is enhanced. These results reinforce previous

Table 2

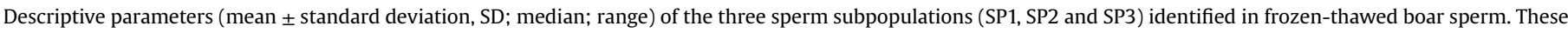
data were obtained after setting the sperm motile subpopulations through principal component and cluster analysis.

\begin{tabular}{|c|c|c|c|c|c|c|c|c|c|}
\hline \multirow{3}{*}{$\mathrm{N}$} & \multicolumn{3}{|l|}{ SP1 } & \multicolumn{3}{|l|}{ SP2 } & \multicolumn{3}{|l|}{ SP3 } \\
\hline & \multicolumn{3}{|l|}{790740} & \multicolumn{3}{|l|}{1295602} & \multicolumn{3}{|l|}{3114629} \\
\hline & mean $\pm S D$ & Median & Range & mean $\pm S D$ & Median & Range & mean $\pm S D$ & Median & Range \\
\hline $\operatorname{VCL}\left(\mu \mathrm{m} \cdot \mathrm{s}^{-1}\right)$ & $67.09 \pm 13.34$ & 64.60 & $46.40-156.60$ & $43.10 \pm 10.20$ & 41.80 & $23.90-93.60$ & $19.39 \pm 7.04$ & 18.20 & $10.00-49.10$ \\
\hline $\operatorname{VSL}\left(\mu \mathrm{m} \cdot \mathrm{s}^{-1}\right)$ & $49.57 \pm 11.96$ & 49.30 & $0.00-90.40$ & $24.13 \pm 9.51$ & 24.40 & $0.00-44.20$ & $6.06 \pm 4.98$ & 4.50 & $0.00-22.30$ \\
\hline $\operatorname{VAP}\left(\mu \mathrm{m} \cdot \mathrm{s}^{-1}\right)$ & $55.71 \pm 9.34$ & 53.95 & $29.50-93.80$ & $32.22 \pm 6.91$ & 31.70 & $14.60-60.40$ & $11.54 \pm 5.42$ & 11.10 & $0.00-29.70$ \\
\hline $\operatorname{LIN}(\%)$ & $75.90 \pm 18.37$ & 81.25 & $0.00-99.00$ & $59.08 \pm 24.91$ & 61.67 & $0.00-98.00$ & $31.61 \pm 23.82$ & 25.65 & $0.00-100.00$ \\
\hline STR (\%) & $88.59 \pm 0.37$ & 93.90 & $0.00-99.00$ & $74.00 \pm 22.23$ & 81.27 & $0.00-100.00$ & $50.02 \pm 27.75$ & 50.72 & $0.00-100.00$ \\
\hline WOB (\%) & $84.53 \pm 12.52$ & 88.47 & $27.00-100.00$ & $76.93 \pm 15.94$ & 79.31 & $22.00-100.00$ & $59.40 \pm 20.82$ & 59.47 & $0.00-100.00$ \\
\hline $\mathrm{ALH}(\mu \mathrm{m})$ & $2.27 \pm 0.02$ & 2.10 & $0.60-7.50$ & $1.99 \pm 0.72$ & 1.90 & $0.40-5.50$ & $1.29 \pm 0.39$ & 1.20 & $0.20-3.20$ \\
\hline $\mathrm{BCF}(\mathrm{Hz})$ & $8.15 \pm 2.50$ & 8.00 & $0.00-16.70$ & $6.24 \pm 2.70$ & 6.00 & $0.00-15.00$ & $2.53 \pm 2.04$ & 2.00 & $0.00-12.00$ \\
\hline
\end{tabular}




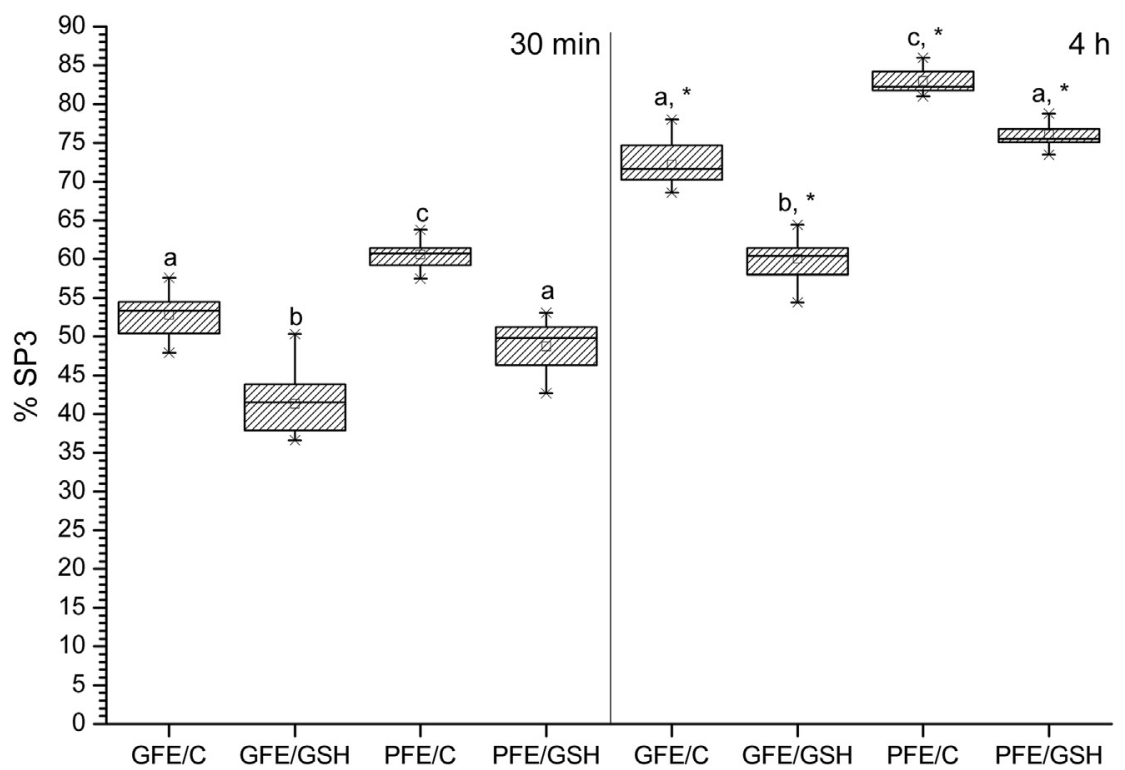

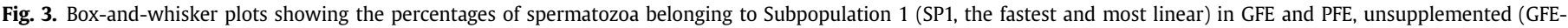

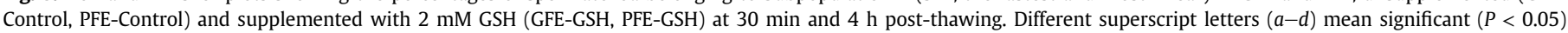

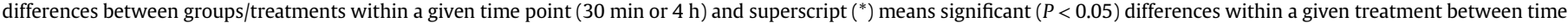
points. GFE: Good freezability ejaculates. PFE: Poor freezability ejaculates. C: Control. GSH: Reduced glutathione (2 mM).

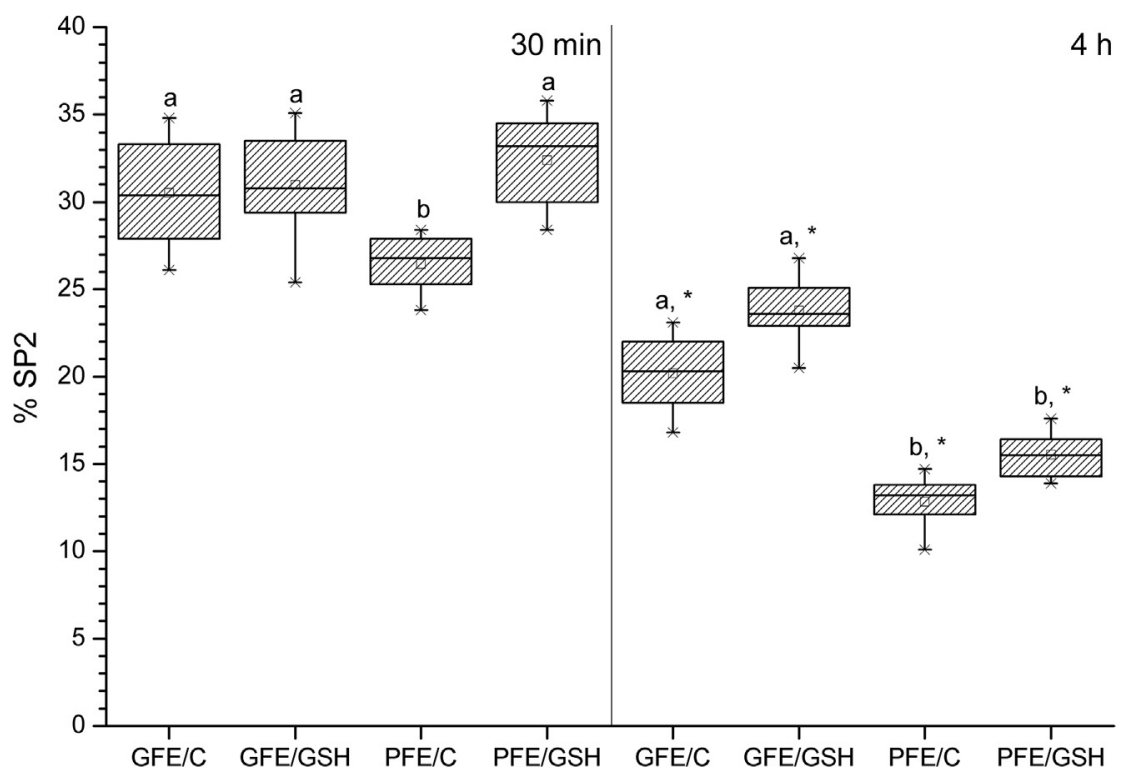

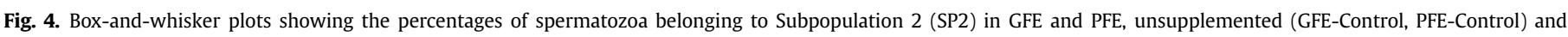

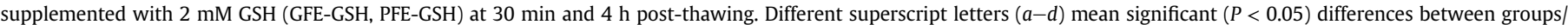

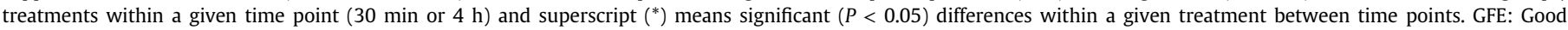
freezability ejaculates. PFE: Poor freezability ejaculates. C: Control. GSH: Reduced glutathione (2 mM).

observations which found that the extent of the cryopreservedinduced decrease in post-thaw sperm motility was lesser when freezing media contained GSH $[6,7,13,15,45]$. In addition, the current work has also observed that PFE, even when freezing media contained GSH, showed significantly lower percentages of membrane-intact and motile spermatozoa than GFE, which also matches with other reports $[7,46,47]$. As boars and ejaculates utilized in this study differed from previous works, these data confirm the positive effects of supplementing freezing media with $2 \mathrm{mM}$ GSH.

Our study has identified three separate subpopulations in boar frozen-thawed sperm. The first subpopulation clustered the fastest and most linear spermatozoa, the second one included those sperm cells with intermediate values and the third subpopulation included the least motile spermatozoa. These data are similar to those obtained in previous reports with liquid-stored and capacitated spermatozoa, suggesting that the presence of three-to-four subpopulations according to kinematic parameters is an inherent characteristic of boar sperm $[2,4,11,12]$. In fact, the detection of specific motile sperm subpopulations in a wide array of mammalian species that are separated with a long phylogenetic distance supports the idea that this structure could play an instrumental role 


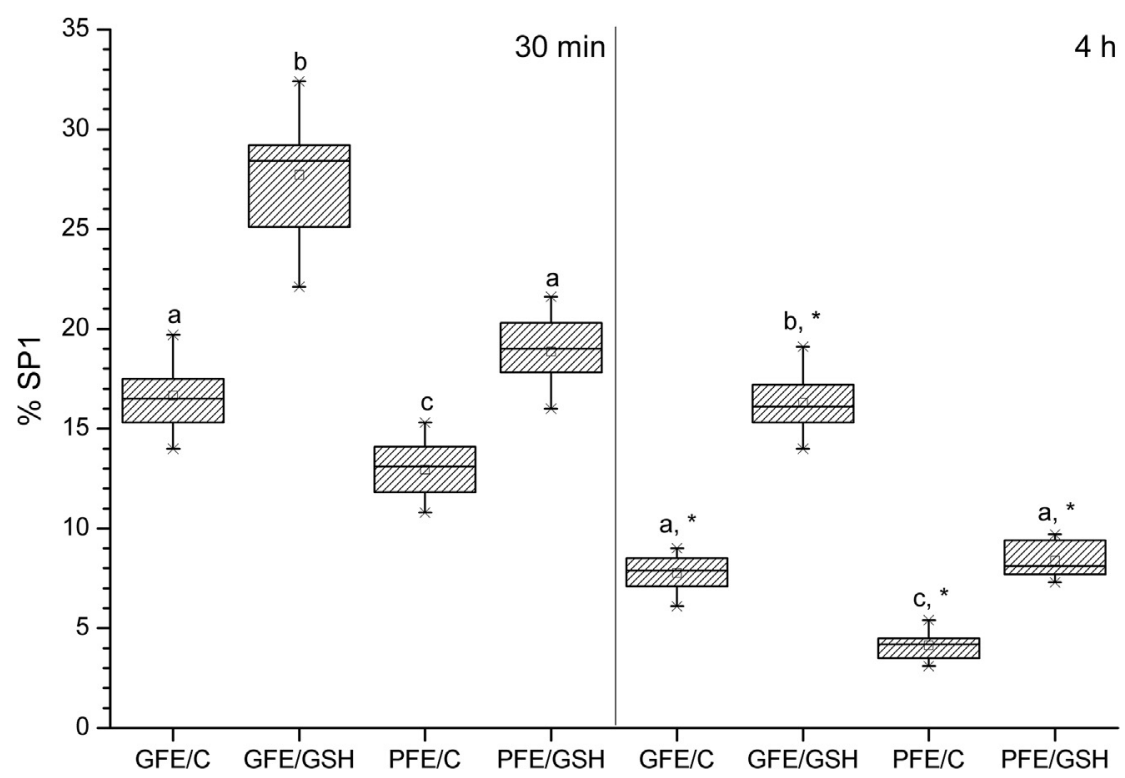

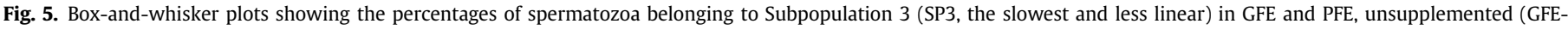

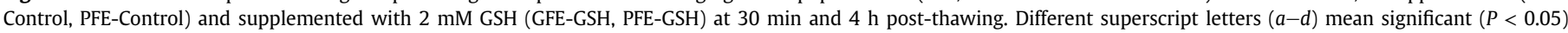

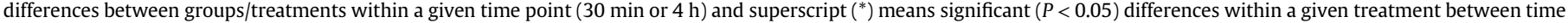
points. GFE: Good freezability ejaculates. PFE: Poor freezability ejaculates. C: Control. GSH: Reduced glutathione (2 mM).

in mammalian sperm function [9,11,19,30,32,34,35]. Yet, this suggestion is sustained by the fact that sperm subpopulations have not only been determined on the basis of kinematic parameters but also on that of morphometry $[28,34,35,41]$.

When subpopulations of GFE and PFE were compared at $30 \mathrm{~min}$ and $4 \mathrm{~h}$ post-thawing, the percentages of SP1 and SP2 were significantly higher in the former than in the latter. Therefore, GFE and PFE do not only differ on the integrity of their sperm membrane and total sperm motility, but also on the distribution of sperm motile subpopulations, which agrees with Flores et al. [12]. As previously mentioned, the addition of GSH to freezing media has previously been reported to increase boar sperm cryotolerance $[13,45,47]$. One of the most interesting effects of supplementing freezing media with GSH is the increase of in vivo fertilizing ability $[6,7]$. Because GSH has been found to increase different sperm parameters in vitro, such as nuclear stability, sperm motility and acrosin activity, it is difficult to ascertain whether there is a particular sperm parameter more affected than others. In fact, while linear regression analyses have demonstrated that the amelioration of nuclear stability (nucleoprotein structure and chromatin integrity) underlies the higher fertilizing ability observed in vivo, correlations between reproductive performance and total and progressive sperm motility are poor [6]. In the current study, we have found that the impact of GSH on sperm motility mainly affects one of the subpopulations, the fastest and most linear one (SP1). There are not many reports evaluating the effects of antioxidants to frozen-thawed semen on motile sperm subpopulations. In a study involving frozen-thawed epididymal red deer sperm, Mata-Campuzano et al. [20] found that the addition of different antioxidants, including $\mathrm{N}$-acetyl-cysteine, dehydroascorbic acid, rutin and Tempol, induced changes in the proportions of sperm belonging to each of the four identified sperm subpopulations.

In the context of the increase in the proportions of SP1spermatozoa observed after adding GSH to freezing media, one should mention two aspects that have been reported by other researchers. On the one hand, the reduced fertility window of frozenthawed spermatozoa compared to fresh/liquid-stored ones, with a maximum interval between insemination and ovulation of $4 \mathrm{~h} \mathrm{[40].}$ Despite not being able to mimic in vivo conditions, the current study observed that the effects of adding GSH to freezing media on motile subpopulations of frozen-thawed sperm were clearly seen in GFE at $4 \mathrm{~h}$ post-thawing, when the proportions of sperm belonging to SP1 were significantly higher in GFE-GSH (mean \pm SEM: $16.3 \pm 0.3$ ) than in the other treatments (GFE-C: $7.8 \pm 0.2$; PFE-C: $4.1 \pm 0.2$; PFE-GSH: $8.4 \pm 0.2$ ).

Another important point to consider is the structure of motile subpopulations and how the increase in the fastest and most linear one, namely SP1, may impact upon reproductive performance. The fact that GSH increases the proportions of sperm belonging to SP1 especially in the case of GFE is a very interesting result, especially if we bear in mind that the fertility window of frozen-thawed sperm is short and that frozen-thawed sperm may encounter difficulties in interacting with oviductal epithelial cells and establishing the sperm reservoir [1]. It is clear that GSH does not exert a particular effect on a discrete sperm parameter but rather maintains the overall structure of sperm cell better, including nuclear and plasma and acrosome membrane integrity $[6,7,15,45]$. However, if frozenthawed boar sperm cryopreserved in the presence of GSH even had difficulties in forming the sperm reservoir, their ability to reach the oviduct and fertilize available oocytes could be related to the intrinsic sperm motility. In this context, the fact that GFE cryopreserved with GSH exhibited higher proportions of SP1, even at $4 \mathrm{~h}$ post-thawing is crucial [40]. Yet, while gravitational force and uterine contractility play a vital role for sperm transport within the sow, the role of intrinsic sperm motility should not be dismissed $[17,18,44]$. At present, no clear relationship has been established between motile subpopulations and reproductive performance in fresh boar semen [31] and, as previously stated, no works studying such a relationship have been conducted with frozen-thawed boar sperm. However, our data on GFE and PFE support the idea that there is a relationship between the sperm subpopulations structure and the resilience to withstand freeze-thawing procedures. Therefore, as the current study has indicated that the presence of GSH induces changes in the structure of subpopulations both in GFE and PFE, it is reasonable to suggest that the effects of GSH on sperm 
motile structure are related to higher cryotolerance. Whether this change in motile subpopulations has a direct impact on reproductive performance through sperm transport or it is rather the increase in ejaculate freezability that explains the main GSH-positive effects remains unknown. Further research is warranted to address to which extent the positive impact of GSH on sperm motility underlies the improvement in reproductive performance.

In conclusion, supplementing freezing media with $2 \mathrm{mM} \mathrm{GSH}$ not only does have a positive impact on the nuclear stability and membrane integrity of boar sperm to withstand freeze-thawing procedures, but the increase in overall sperm motility at postthawing reported in previous studies is related to the structure of sperm subpopulations. Further research is required to address whether the higher proportions of sperm belonging to SP1 are related to the increase in reproductive performance mediated by GSH and observed in previous studies.

\section{Acknowledgements}

The authors acknowledge the support from Ministry of Economy and Competitiveness, Spain (Grants: AGL-2008-01792/GAN, JCI-2010-08620 and RYC-2014-15581).

\section{Appendix A. Supplementary data}

Supplementary data related to this article can be found at http:// dx.doi.org/10.1016/j.cryobiol.2017.07.002.

\section{References}

[1] M. Abad, D.J. Sprecher, P. Ross, R.M. Friendship, R.N, Effect of sperm cryopreservation and supplementing semen doses with seminal plasma on the establishment of a sperm reservoir in gilts, Reprod. Domest. Anim. 42 (2007) 149-152.

[2] T. Abaigar, W.V. Holt, R.A. Harrison, G. del Barrio, Sperm subpopulations in boar (Sus scrofa) and gazelle (Gazella dama mhorr) semen as revealed by pattern analysis of computer-assisted motility assessments, Biol. Reprod. 60 (1999) 32-41.

[3] I. Casas, E. Torner, M. Yeste, S. Bonet S, Boar sperm thawing practices: the number of straws does matter, Theriogenology 77 (2012) 1487-1494.

[4] T. Cremades, J. Roca, H. Rodriguez-Martinez, T. Abaigar, J.M. Vazquez, E.A. Martinez, Kinematic changes during the cryopreservation of boar spermatozoa, J. Androl. 26 (2005) 610-618.

[5] J. Dorado, D. Acha, I. Ortiz, M.J. Gálvez, J.J. Carrasco, B. Díaz, V. Gómez-Arrones, R. Calero-Carretero, M. Hidalgo, Relationship between conventional semen characteristics, sperm motility patterns and fertility of Andalusian donkeys (Equus asinus), Anim. Reprod. Sci. 143 (2013) 64-71.

[6] E. Estrada, J.E. Rodríguez-Gil, L.G. Rocha, S. Balasch, S. Bonet, M. Yeste, Supplementing cryopreservation media with reduced glutathione increases fertility and prolificacy of sows inseminated with frozen-thawed boar semen, Andrology 2 (2014) 88-99.

[7] E. Estrada, J.E. Rodríguez-Gil, M.M. Rivera del Álamo, A. Peña, M. Yeste, Effects of reduced glutathione on acrosin activity in frozen-thawed boar spermatozoa, Reprod. Fertil. Dev. (2015), http://dx.doi.org/10.1071/RD15118.

[8] R. Fernández-Gago, M. Álvarez-Rodríguez, M.E. Alonso, J.R. González, B. Alegre, J.C. Domínguez, F. Martínez-Pastor, Thawing boar semen in the presence of seminal plasma improves motility, modifies subpopulation patterns and reduces chromatin alterations, Reprod. Fertil. Dev. (2016), http:// dx.doi.org/10.1071/RD15530.

[9] M.A. Ferraz, R. Morató, M. Yeste, N. Arcarons, A.I. Pena, C. Tamargo, C.O. Hidalgo, R. Muiño, T. Mogas, Evaluation of sperm subpopulation structure in relation to in vitro sperm-oocyte interaction of frozen-thawed semen from Holstein bulls, Theriogenology 81 (2014) 1067-1072.

[10] P.S. Fiser, R.W. Fairfull, C. Hansen, P.L. Panich, J.N. Shrestha, L. Underhill, The effect of warming velocity on motility and acrosomal integrity of boar sperm as influenced by the rate of freezing and glycerol level, Mol. Reprod. Dev. 34 (1993) 190-195.

[11] E. Flores, E. Taberner, M.M. Rivera, A. Peña, T. Rigau, J. Miró, J.E. Rodríguez-Gil Effects of freezing/thawing on motile sperm subpopulations of boar and donkey ejaculates, Theriogenology 70 (2008) 936-945.

[12] E. Flores, J.M. Fernández-Novell, A. Peña, J.E. Rodríguez-Gil, The degree of resistance to freezing-thawing is related to specific changes in the structures of motile sperm subpopulations and mitochondrial activity in boar spermatozoa, Theriogenology 72 (2009) 784-797.

[13] J. Gadea, F.A. García-Vázquez, C. Matás, J.C. Gardón, S. Cánovas, D. Gumbao,
Cooling and freezing of boar spermatozoa: supplementation of the freezing media with reduced glutathione preserves sperm function, J. Androl. 26 (2005) 396-404

[14] D.L. Garner, L.A. Johnson, Viability assessment of mammalian sperm using SYBR-14 and propidium iodide, Biol. Reprod. 53 (1995) 276-284.

[15] E. Giaretta, E. Estrada, D. Bucci, M. Spinaci, J.E. Rodríguez-Gil, M. Yeste, Combining reduced glutathione and ascorbic acid has supplementary beneficial effects on boar sperm cryotolerance, Theriogenology 83 (2015) 399-407.

[16] J.L. Hancock, G.J.L. Howell, The collection of boar semen, Vet. Rec. 71 (1959) 664-665.

[17] P. Langendijk, E.G. Bouwman, A. Kidson, R.N. Kirkwood, N.M. Soede, B. Kemp, Functions of myometrial activity in sperm transport through the genital tract and fertilisation in sows, Reproduction 123 (2002) 683-690.

[18] P. Langendijk, N.M. Soede, B. Kemp B, Uterine activity, sperm transport, and the role of boar stimuli around insemination in sows, Theriogenology 63 (2005) 500-513.

[19] C. Luna, M. Yeste, M.M. Rivera Del Alamo, J. Domingo, A. Casao, J.E. RodriguezGil, R. Pérez-Pé, J.A. Cebrián-Pérez, T. Muiño-Blanco, Effect of seminal plasma proteins on the motile sperm subpopulations in ram ejaculates, Reprod. Fertil. Dev. (2015), http://dx.doi.org/10.1071/RD15231.

[20] M. Mata-Campuzano, M. Alvarez-Rodríguez, E. del Olmo, M.R. FernándezSantos, J.J. Garde, F. Martínez-Pastor, Quality, oxidative markers and DNA damage (DNA) fragmentation of red deer thawed spermatozoa after incubation at $37{ }^{\circ} \mathrm{C}$ in presence of several antioxidants, Theriogenology 78 (2012) 1005-1019.

[21] M.J. Maya-Soriano, E. Taberner, M. Sabés-Alsina, J. Ramon, O. Rafel, L. Tusell, M. Piles, M. López-Béjar, Daily exposure to summer temperatures affects the motile subpopulation structure of epididymal sperm cells but not male fertility in an in vivo rabbit model, Theriogenology 84 (2015) 384-389.

[22] J. Miró, E. Taberner, M. Rivera, A. Peña, A. Medrano, T. Rigau, A. Peñalba, Effects of dilution and centrifugation on the survival of spermatozoa and the structure of motile sperm cell subpopulations in refrigerated Catalonian donkey semen, Theriogenology 72 (2009) 1017-1022.

[23] M.T. Mogas, M.R. Alamo, J.E. Rodríguez-Gil, Roles of $\mathrm{Na}(+) / \mathrm{K}(+)$-dependent ATPase, $\mathrm{Na}(+) / \mathrm{H}(+)$ antiporter and GLUT hexose transporters in the cryosurvival of dog spermatozoa: effects on viability, acrosome state and motile sperm subpopulation structure, Theriogenology 75 (2011) 1669-1681.

[24] S.T. Mortimer, CASA - practical aspects, J. Androl. 21 (2000) 515-524.

[25] R. Muiño, M.M. Rivera, T. Rigau, J.E. Rodriguez-Gil, A.I. Peña, Effect of different thawing rates on post-thaw sperm viability, kinematic parameters and motile sperm subpopulations structure of bull semen, Anim. Reprod. Sci. 109 (2008) 50-64.

[26] C. Ortega-Ferrusola, B. Macías García, V. Suárez Rama, J.M. Gallardo-Bolaños, L. González-Fernández, J.A. Tapia, H. Rodríguez-Martinez, F.J. Peña, Identification of sperm subpopulations in stallion ejaculates: changes after cryopreservation and comparison with traditional statistics, Reprod. Domest. Anim. 44 (2009) 419-423.

[27] A.I. Peña, M. Barrio, J.J. Becerra, L.A. Quintela, P.G. Herradón, Motile sperm subpopulations in frozen-thawed dog semen: changes after incubation in capacitating conditions and relationship with sperm survival after osmotic stress, Anim. Reprod. Sci. 133 (2012) 214-223.

[28] F.J. Peña, F. Saravia, M. García-Herreros, I. Núñez-Martínez, J.A. Tapia, A. Johannisson, M. Wallgren, H. Rodríguez-Martínez, Identification of sperm morphometric subpopulations in two different portions of the boar ejaculate and its relation to postthaw quality, J. Androl. 26 (2005) 716-723.

[29] V.G. Pursel, L.A. Johnson LA, Freezing of boar spermatozoa: fertilizing capacity with concentrated semen and a new thawing procedure, J. Anim. Sci. 40 (1975) 99-102.

[30] A. Quintero-Moreno, J. Miró, A. Teresa Rigau, J.E. Rodríguez-Gil, Identification of sperm subpopulations with specific motility characteristics in stallion ejaculates, Theriogenology 59 (2003) 1973-1990.

[31] A. Quintero-Moreno, T. Rigau, J.E. Rodríguez-Gil, Regression analyses and motile sperm subpopulation structure study as improving tools in boar semen quality analysis, Theriogenology 61 (2004) 673-690.

[32] A. Quintero-Moreno, T. Rigau, J.E. Rodríguez-Gil, Multivariate cluster analysis regression procedures as tools to identify motile sperm subpopulations in rabbit semen and to predict semen fertility and litter size, Reprod. Domest. Anim. 42 (2007) 312-319.

[33] J.E. Rodríguez-Gil, Biological aspects of the mature boar spermatozoon, in: S. Bonet, I. Casas, W.V. Holt, M. Yeste (Eds.), Boar Reproduction: Fundamentals and New Biotechnological Trends, Springer, Berlin, 2013, pp. 49-64.

[34] P. Santolaria, S. Vicente-Fiel, I. Palacín, E. Fantova, M.E. Blasco, M.A. Silvestre, J.L. Yániz, Predictive capacity of sperm quality parameters and sperm subpopulations on field fertility after artificial insemination in sheep, Anim. Reprod. Sci. 163 (2015) 82-88.

[35] L.M. Thurston, P.F. Watson, W.V. Holt, Sources of variation in the morphological characteristics of sperm subpopulations assessed objectively by a novel automated sperm morphology analysis system, J. Reprod. Fertil. 117 (1999) $271-280$.

[36] L.M. Thurston, P.F. Watson, A.J. Mileham, W.V. Holt, Morphologically distinct sperm subpopulations defined by Fourier shape descriptors in fresh ejaculates correlate with variation in boar semen quality following cryopreservation, J. Androl. 22 (2001) 382-394.

[37] L.M. Thurston, K. Siggins, A.J. Mileham, P.F. Watson, W.V. Holt, Identification of 
amplified restriction fragment length polymorphism markers linked to genes controlling boar sperm viability following cryopreservation, Biol. Reprod. 66 (2002a) 545-554.

[38] L.M. Thurston, P.F. Watson, W.V. Holt, Semen cryopreservation: a genetic explanation for species and individual variation? Cryo Lett. 23 (2002b) 255-262.

[39] A.J. Vázquez, M.J. Cedillo, V.J. Quezada, A.C. Rivas, E.C. Morales, E.M. Ayala, M.J. Hernández, R.A. González, M.A. Aragón, Effects of repeated electroejaculations on kinematic sperm subpopulations and quality markers of Mexican creole goats, Anim. Reprod. Sci. 154 (2015) 29-38.

[40] D. Waberski, K.F. Weitze, T. Gleumes, M. Schwarz, T. Willmen, R. Petzoldt, Effect of time of insemination relative to ovulation on fertility with liquid and frozen boar semen, Theriogenology 42 (1994) 831-840.

[41] J.L. Yániz, I. Palacín, S. Vicente-Fiel, J.A. Sánchez-Nadal, P. Santolaria, Sperm population structure in high and low field fertility rams, Anim. Reprod. Sci. 156 (2015) 128-134.

[42] M. Yeste, Recent advances in boar sperm cryopreservation: state of the art and current perspectives, Reprod. Domest. Anim. 50 (Suppl 2) (2015) 71-79.

[43] M. Yeste, Sperm cryopreservation update: cryodamage, markers and factors affecting the sperm freezability in pigs, Theriogenology 85 (2016) 47-64.
[44] M. Yeste, M. Castillo-Martín, Boar spermatozoa within the Uterus, in: S. Bonet, I. Casas, W.V. Holt, M. Yeste (Eds.), Boar Reproduction: Fundamentals and New Biotechnological Trends, Springer, Berlin, 2013, pp. 101-168.

[45] M. Yeste, E. Flores, E. Estrada, S. Bonet, T. Rigau, J.E. Rodríguez-Gil, Reduced glutathione and procaine hydrochloride protect the nucleoprotein structure of boar spermatozoa during freeze-thawing by stabilising disulphide bonds, Reprod. Fertil. Dev. 25 (2013a) 1036-1050.

[46] M. Yeste, E. Estrada, I. Casas, S. Bonet, J.E. Rodríguez-Gil, Good and bad freezability boar ejaculates differ in the integrity of nucleoprotein structure after freeze-thawing but not in ROS levels, Theriogenology 79 (2013b) 929-939.

[47] M. Yeste, E. Estrada, E. Pinart, S. Bonet, J. Miró, J.E. Rodríguez-Gil, The improving effect of reduced glutathione on boar sperm cryotolerance is related with the intrinsic ejaculate freezability, Cryobiology 68 (2014a) $251-261$.

[48] M. Yeste, E. Estrada, M.M. Rivera, S. Bonet, T. Rigau, J.E. Rodríguez-Gil, The increase in phosphorylation levels of serine residues of protein HSP70 during holding time at $17{ }^{\circ} \mathrm{C}$ is concomitant with a higher cryotolerance of boar spermatozoa, PLoS One 9 (2014b) e90887. 